\title{
On Talagrand's Admissible Net Approach to Majorizing Measures and Boundedness of Stochastic Processes \\ by
}

\author{
Witold BEDNORZ \\ Presented by Stanistaw KWAPIEN
}

Summary. We show that the main result of [1] on sufficiency of existence of a majorizing measure for boundedness of a stochastic process can be naturally split in two theorems, each of independent interest. The first is that the existence of a majorizing measure is sufficient for the existence of a sequence of admissible nets (as recently introduced by Talagrand [5]), and the second that the existence of a sequence of admissible nets is sufficient for sample boundedness of a stochastic process with bounded increments.

1. Introduction. Let $(T, d)$ be a compact metric space, and let $\varphi$ : $\mathbb{R}_{+} \rightarrow \mathbb{R}_{+}$be a Young function, i.e. convex, increasing, continuous and such that $\varphi(0)=0$. We say that a stochastic process $X(t), t \in T$, has bounded increments if

$$
\mathbf{E} \varphi\left(\frac{|X(s)-X(t)|}{d(s, t)}\right) \leq 1 \quad \text { for } s, t \in T,
$$

Without losing generality one can assume that $\varphi$ is normalized, i.e. $\varphi(1)=1$. Note that under (1) there exists a separable modification of $X(t), t \in T$, which we always refer to when considering a process with bounded increments.

We say that a Borel probability measure $m$ on $(T, d)$ is majorizing if

$$
\mathcal{M}(m, \varphi):=\sup _{t \in T} \int_{0}^{D(t, T)} \varphi^{-1}\left(\frac{1}{m(B(t, \varepsilon))}\right) d \varepsilon<\infty,
$$

2000 Mathematics Subject Classification: 60G17, 28A99.

Key words and phrases: majorizing measure, sample boundedness.

Partially supported by Grant MENiN 1 P03A 01229. 
and weakly majorizing if

$$
\overline{\mathcal{M}}(m, \varphi):=\int_{T} \int_{0}^{D(t, T)} \varphi^{-1}\left(\frac{1}{m(B(t, \varepsilon))}\right) d \varepsilon m(d t)<\infty,
$$

where $B(t, \varepsilon):=\{s \in T: d(s, t) \leq \varepsilon\}$ and $D(t, T):=\sup \{d(s, t): s \in T\}$. The concept of majorizing measure was introduced by Fernique [2] for the purpose of proving boundedness of stochastic processes. For the historical background on the sample boundedness of stochastic processes under the bounded increment assumption we refer to [2], [3] and [5]. The following theorem proved in [1] is a generalization of Fernique's result as well as Talagrand's:

THEOREM 1. If $\varphi$ is a Young function and $m$ a majorizing measure on $T$ then, for each separable stochastic process $X(t), t \in T$, which satisfies (1),

$$
\mathbf{E} \sup _{s, t \in T}|X(s)-X(t)| \leq 32 \mathcal{M}(m, \varphi) .
$$

In this paper we pursue a new approach to Theorem 2 using the language of admissible nets (cf. Definition 1.2.3 in [3]). Below we give a definition of admissible nets suitable for our purposes. Let $\left(N_{k}\right)_{k \geq 0}$ be a sequence of positive reals such that $N_{0}=1$ and

$$
c \varphi^{-1}\left(N_{k}\right) \leq \varphi^{-1}\left(N_{k+1}\right) \leq C \varphi^{-1}\left(N_{k}\right) \quad \text { for } k \geq 1,
$$

where $2<c \leq C$ (the usual choice is $N_{k}:=\varphi\left(R^{k}\right)$, where $R>2$ ). We will say that $\mathcal{T}:=\left(T_{k}\right)_{k \geq 0}$ is an admissible sequence of nets if $\left|T_{k}\right| \leq N_{k}$ and

$$
\begin{aligned}
& \mathcal{A}(\mathcal{T}, \varphi):=\sup _{u \in T} \sum_{k=0}^{\infty} d\left(u, T_{k}\right) \varphi^{-1}\left(N_{k}\right)<\infty, \\
& \overline{\mathcal{A}}(\mathcal{T}, \varphi):=\sum_{k=0}^{\infty} \sum_{u \in T_{k+1}} \frac{d\left(u, T_{k}\right) \varphi^{-1}\left(N_{k}\right)}{N_{k+1}}<\infty .
\end{aligned}
$$

Theorem 1 can be obtained as a corollary of the following two theorems, which are of independent interest:

TheOREM 2. For each sequence of admissible nets $\mathcal{T}=\left(T_{k}\right)_{k \geq 0}$ and any stochastic process $X(t), t \in T$, satisfying (1),

$$
\mathbf{E} \sup _{s, t \in T}|X(s)-X(t)| \leq \frac{4 c C}{c-2} \mathcal{A}(\mathcal{T}, \varphi)+2 C \overline{\mathcal{A}}(\mathcal{T}, \varphi) .
$$

THEOREM 3. If $(T, d)$ admits a majorizing measure $m$ then there exists a sequence of nets $\mathcal{T}=\left(T_{k}\right)_{k \geq 0}$ such that $\left|T_{k}\right| \leq N_{k}$ for $k \geq 0$ and

$$
\mathcal{A}(\mathcal{T}, \varphi) \leq \frac{4 c}{c-1} \mathcal{M}(\mathcal{T}, \varphi), \quad \overline{\mathcal{A}}(\mathcal{T}, \varphi) \leq \frac{4 c}{c-1} \overline{\mathcal{M}}(m, \varphi) .
$$


Indeed, since clearly $\overline{\mathcal{M}}(m, \varphi) \leq \mathcal{M}(m, \varphi)$, Theorems 2 and 3 show that the existence of a majorizing measure implies the sample boundedness of any stochastic process with bounded increments, so in this way we reprove Theorem 1.

2. Sample boundedness via admissible nets. Let $\pi_{k}(t)$ be any point in $T_{k}$ which satisfies $d\left(t, T_{k}\right)=d\left(t, \pi_{k}(t)\right)$, i.e. a point in $T_{k}$ closest to $t$.

Proof of Theorem 2. Fix $l \geq 0$ and $t \in T$. Clearly one may assume that $\lim _{k \rightarrow \infty} d\left(t, T_{k}\right)=0$ since otherwise the right hand side in (4) is infinite and there is nothing to prove. We define $t_{l}=\pi_{l}(t)$ and by reverse induction, $t_{k}=\pi_{k}\left(t_{k+1}\right)$. By the chain argument we obtain

$$
\left|f\left(t_{l}\right)-f\left(t_{0}\right)\right| \leq \sum_{j=0}^{l-1}\left|f\left(t_{j}\right)-f\left(t_{j+1}\right)\right| .
$$

For all Young functions $\varphi$ we clearly have

$$
\frac{x}{y} \leq 1+\frac{\varphi(x)}{\varphi(y)}, \quad x, y>0 .
$$

Setting $x=\left|f\left(t_{j}\right)-f\left(t_{j+1}\right)\right| / d\left(t_{j}, t_{j+1}\right)$ and $y=\varphi^{-1}\left(N_{j+1}\right)$ in (6), we derive that

$$
\frac{\left|f\left(t_{j}\right)-f\left(t_{j+1}\right)\right|}{d\left(t_{j}, t_{j+1}\right) \varphi^{-1}\left(N_{j+1}\right)} \leq 1+\frac{1}{N_{j+1}} \varphi\left(\frac{\left|f\left(t_{j}\right)-f\left(t_{j+1}\right)\right|}{d\left(t_{j}, t_{j+1}\right)}\right) .
$$

Since by (3) we have $\varphi^{-1}\left(N_{j+1}\right) \leq C \varphi^{-1}\left(N_{j}\right)$, we can see that

$$
\left|f\left(t_{j}\right)-f\left(t_{j+1}\right)\right| \leq C d\left(t_{j}, t_{j+1}\right) \varphi^{-1}\left(N_{j}\right)\left(1+\frac{1}{N_{j+1}} \varphi\left(\frac{\left|f\left(t_{j}\right)-f\left(t_{j+1}\right)\right|}{d\left(t_{j}, t_{j+1}\right)}\right)\right) .
$$

This implies that

$$
\begin{aligned}
\mid f\left(t_{l}\right)- & f\left(t_{0}\right) \mid \\
\leq & C \sum_{j=0}^{l-1} d\left(t_{j}, t_{j+1}\right) \varphi^{-1}\left(N_{j}\right) \\
& +C \sum_{k=0}^{\infty} \sum_{u \in T_{k+1}} \frac{d\left(u, T_{k}\right) \varphi^{-1}\left(N_{k}\right)}{N_{k+1}} \varphi\left(\frac{\left|f(u)-f\left(\pi_{k}(u)\right)\right|}{d\left(u, \pi_{k}(u)\right)}\right) .
\end{aligned}
$$

LEMma 1. The following inequality holds:

$$
\sum_{j=0}^{l-1} d\left(t_{j}, t_{j+1}\right) \varphi^{-1}\left(N_{j}\right) \leq \frac{2 c}{c-2} \sum_{k=0}^{l} d\left(t, \pi_{k}(t)\right) \varphi^{-1}\left(N_{k}\right) .
$$


Proof. We first show that for each $0 \leq j \leq l$ we have

$$
d\left(t, t_{j}\right) \varphi^{-1}\left(N_{j}\right) \leq \sum_{k=j}^{l}\left(\frac{2}{c}\right)^{j-k} d\left(t, T_{k}\right) \varphi^{-1}\left(N_{k}\right),
$$

where $c$ is the constant in (3). The proof goes by reverse induction. The case $j=l$ is trivial, so we may assume that

$$
d\left(t, t_{j+1}\right) \varphi^{-1}\left(N_{j+1}\right) \leq \sum_{k=j+1}^{l}\left(\frac{2}{c}\right)^{j+1-k} d\left(t, T_{k}\right) \varphi^{-1}\left(N_{k}\right) .
$$

Note that the definition of $\pi_{j}$ implies that

$$
d\left(t_{j}, t_{j+1}\right)=d\left(\pi_{j}\left(t_{j+1}\right), t_{j+1}\right) \leq d\left(\pi_{j}(t), t_{j+1}\right) \leq d\left(t, \pi_{j}(t)\right)+d\left(t, t_{j+1}\right),
$$

which combined with $d\left(t, t_{j}\right) \leq d\left(t, t_{j+1}\right)+d\left(t_{j}, t_{j+1}\right)$ results in

$$
d\left(t, t_{j}\right) \leq d\left(t, \pi_{j}(t)\right)+2 d\left(t, t_{j+1}\right) .
$$

From (3) we obtain

$$
\begin{aligned}
d\left(t, t_{j}\right) \varphi^{-1}\left(N_{j}\right) & \leq\left(d\left(t, \pi_{j}(t)\right)+2 d\left(t, \pi_{j+1}(t)\right)\right) \varphi^{-1}\left(N_{j}\right) \\
& \leq d\left(t, \pi_{j}(t)\right) \varphi^{-1}\left(N_{j}\right)+\frac{2}{c} d\left(t, \pi_{j+1}(t)\right) \varphi^{-1}\left(N_{j+1}\right) .
\end{aligned}
$$

The induction assumption (9) now yields (8). We finish the proof of the lemma by first checking that

$$
\sum_{j=0}^{l-1} d\left(t_{j}, t_{j+1}\right) \varphi^{-1}\left(N_{j}\right) \leq 2 \sum_{j=0}^{l} d\left(t, t_{j}\right) \varphi^{-1}\left(N_{j}\right)
$$

and then applying (8) so that

$$
\begin{aligned}
\sum_{j=0}^{l} d\left(t, t_{j}\right) \varphi^{-1}\left(N_{j}\right) & \leq \sum_{j=0}^{l}\left(\sum_{k=j}^{l}\left(\frac{c}{2}\right)^{j-k} d\left(t, \pi_{k}(t)\right) \varphi^{-1}\left(N_{k}\right)\right) \\
& \leq \sum_{k=0}^{l}\left(\sum_{j=k}^{l}\left(\frac{c}{2}\right)^{j-k}\right) d\left(t, \pi_{k}(t)\right) \varphi^{-1}\left(N_{k}\right) \\
& \leq \frac{c}{c-2} \sum_{k=0}^{l} d\left(t, \pi_{k}(t)\right) \varphi^{-1}\left(N_{k}\right) .
\end{aligned}
$$

We use (7) and Lemma 1 to show that

$$
\begin{aligned}
\left|f\left(t_{l}\right)-f\left(t_{0}\right)\right| \leq & \frac{2 c C}{c-2} \sum_{k=0}^{l} d\left(t, T_{k}\right) \varphi^{-1}\left(N_{k}\right) \\
& +C \sum_{k=0}^{\infty} \sum_{u \in T_{k+1}} \frac{d\left(u, T_{k}\right) \varphi^{-1}\left(N_{k}\right)}{N_{k+1}} \varphi\left(\frac{\left|f(u)-f\left(\pi_{k}(u)\right)\right|}{d\left(u, \pi_{k}(u)\right)}\right) .
\end{aligned}
$$


From the property $\lim _{k \rightarrow \infty} d\left(t, T_{k}\right)=0$ we deduce that

$$
\begin{aligned}
\left|f(t)-f\left(t_{0}\right)\right| \leq & \frac{4 c C}{c-2} \sup _{u \in T} \sum_{k=0}^{\infty} d\left(u, T_{k}\right) \varphi^{-1}\left(N_{k}\right) \\
& +2 C \sum_{k=0}^{\infty} \sum_{u \in T_{k+1}} \frac{d\left(u, T_{k}\right) \varphi^{-1}\left(N_{k}\right)}{N_{k+1}} \varphi\left(\frac{\left|f(u)-f\left(\pi_{k}(u)\right)\right|}{d\left(u, \pi_{k}(u)\right)}\right) .
\end{aligned}
$$

Since $t_{0}=\pi_{0}(T)$ is the only point in $T_{0}$ which does not depend on $t$, it is clear that for any $s, t \in T$ we have

$$
\begin{aligned}
\mid f(s)- & f(t) \mid \\
\leq & \frac{4 c C}{c-2} \sup _{u \in T} \sum_{k=0}^{\infty} d\left(u, T_{k}\right) \varphi^{-1}\left(N_{k}\right) \\
& \quad+2 C \sum_{k=0}^{\infty} \sum_{u \in T_{k+1}} \frac{d\left(u, T_{k}\right) \varphi^{-1}\left(N_{k}\right)}{N_{k+1}} \varphi\left(\frac{\left|f(u)-f\left(\pi_{k}(u)\right)\right|}{d\left(u, \pi_{k}(u)\right)}\right) .
\end{aligned}
$$

Having thus established the result for any continuous functions $f$ on $(T, d)$ we turn to its stochastic version. By a standard argument (see Theorem 2.3 of [3] or Theorem 3.1 of [1]) it suffices to prove Theorem 2 for processes with a.s. Lipschitz samples (with respect to $d$ ). By the Fubini theorem and (1) we obtain

$$
\begin{aligned}
\mathbf{E} \sup _{s, t \in T} & |X(s)-X(t)| \leq \frac{4 c C}{c-2} \sup _{u \in T} \sum_{k=0}^{\infty} d\left(u, T_{k}\right) \varphi^{-1}\left(N_{k}\right) \\
& +2 C \sum_{k=0}^{\infty} \sum_{u \in T_{k+1}} \frac{d\left(u, T_{k}\right) \varphi^{-1}\left(N_{k}\right)}{N_{k+1}} \mathbf{E} \varphi\left(\frac{\left|f(u)-f\left(\pi_{k}(u)\right)\right|}{d\left(u, \pi_{k}(u)\right)}\right) \\
\leq & \frac{4 c C}{c-2} \sup _{u \in T} \sum_{k=0}^{\infty} d\left(u, T_{k}\right) \varphi^{-1}\left(N_{k}\right)+2 C \sum_{k=0}^{\infty} \sum_{u \in T_{k+1}} \frac{d\left(u, T_{k}\right) \varphi^{-1}\left(N_{k}\right)}{N_{k+1}}
\end{aligned}
$$

3. Construction of a sequence of admissible nets. We describe how to construct a sequence of admissible nets when we have a majorizing measure $m$ on $(T, d)$ (thus in particular $\operatorname{supp}(m)=T$ ). Let

$$
r_{k}(t):=\inf \left\{\varepsilon>0: m(B(t, \varepsilon)) \geq 1 / N_{k}\right\},
$$

where $\left(N_{k}\right)_{k \geq 0}$ satisfies (3). Clearly $m\left(B\left(t, r_{k}(t)\right)\right) \geq 1 / N_{k}$ and $r_{0}(t) \leq$ $D(t, T)$. In [1] two simple properties of $r_{k}$ are given; we repeat their proofs for completeness.

Lemma 2. The functions $r_{k}, k \geq 0$, are 1-Lipschitz for all $t \in T$. 
Proof. A geometrical argument shows that

$$
B\left(s, r_{k}(t)+d(s, t)\right) \supset B\left(t, r_{k}(t)\right),
$$

and consequently $m\left(B\left(s, r_{k}(t)+d(s, t)\right)\right) \geq 1 / N_{k}$. Hence $r_{k}(s) \leq r_{k}(t)+$ $d(s, t)$ and similarly $r_{k}(t) \leq r_{k}(s)+d(s, t)$, which implies that $r_{k}$ is 1Lipschitz.

Lemma 3. For each $0 \leq \delta \leq D(t, T)$ we have

$$
\sum_{k=0}^{\infty} \min \left\{r_{k}(t), \delta\right\} \varphi^{-1}\left(N_{k}\right) \leq \frac{c}{c-1} \int_{0}^{\delta} \varphi^{-1}\left(\frac{1}{m(B(t, \varepsilon))}\right) d \varepsilon .
$$

Proof. Observe that there exists $k_{0} \geq 0$ such that $r_{k_{0}+1}(t)<\delta \leq r_{k_{0}}(t)$. Clearly

$$
\int_{r_{k+1}(t)}^{r_{k}(t)} \varphi^{-1}\left(\frac{1}{m(B(t, \varepsilon))}\right) d \varepsilon \geq\left(r_{k}(t)-r_{k+1}(t)\right) \varphi^{-1}\left(N_{k}\right),
$$

and in the same way we show that

$$
\int_{r_{k_{0}+1}(t)}^{\delta} \varphi^{-1}\left(\frac{1}{m(B(t, \varepsilon))}\right) d \varepsilon \geq\left(\delta-r_{k_{0}+1}(t)\right) \varphi^{-1}\left(N_{k_{0}}\right) .
$$

Thus using (3) we deduce that

$$
\begin{aligned}
& \int_{0}^{\delta} \varphi^{-1}\left(\frac{1}{m(B(t, \varepsilon))}\right) d \varepsilon \\
& \geq \sum_{k=k_{0}+1}^{\infty}\left(r_{k}(t)-r_{k+1}(t)\right) \varphi^{-1}\left(N_{k}\right)+\left(\delta-r_{k_{0}+1}(t)\right) \varphi^{-1}\left(N_{k_{0}}\right) \\
& \geq \sum_{k=k_{0}+1}^{\infty} r_{k}(t)\left(\varphi^{-1}\left(N_{k}\right)-\varphi^{-1}\left(N_{k-1}\right)\right)+\delta \varphi^{-1}\left(N_{k_{0}}\right) \\
& \geq \frac{c-1}{c} \sum_{k=k_{0}+1}^{\infty} r_{k}(t) \varphi^{-1}\left(N_{k}\right)+\delta \varphi^{-1}\left(N_{k_{0}}\right) .
\end{aligned}
$$

Since

$$
\sum_{k=0}^{k_{0}} \varphi^{-1}\left(N_{k}\right) \leq \sum_{k=0}^{k_{0}} c^{-k} \varphi^{-1}\left(N_{k_{0}}\right) \leq \frac{c}{c-1} \varphi^{-1}\left(N_{k_{0}}\right)
$$

we finally obtain

$$
\int_{0}^{\delta} \varphi^{-1}\left(\frac{1}{m(B(t, \varepsilon))}\right) d \varepsilon \geq \frac{c-1}{c} \sum_{k=0}^{\infty} \min \left\{r_{k}(t), \delta\right\} \varphi^{-1}\left(N_{k}\right) .
$$


The construction of a sequence of admissible nets $\mathcal{T}=\left(T_{k}\right)_{k \geq 0}$, assuming the existence of a majorizing measure, is based on the following intermediate result:

THEOREM 4. There exists a sequence of nets $\mathcal{T}=\left(T_{k}\right)_{k \geq 0}, T_{k} \subset T$, that satisfies the following conditions:

1. $\left|T_{0}\right|=1,\left|T_{k}\right| \leq N_{k}$;

2. $B\left(t, r_{k}(t)\right)$ are disjoint for $t \in T_{k}$;

3. for each $t \in T$ we have $d\left(t, T_{k}\right) \leq 4 r_{k}(t)$;

4. $r_{k}(t) \leq 2 r_{k}(x)$ for each $t \in T_{k}$ and $x \in B\left(t, r_{k}(t)\right)$.

Proof. Fix $k \geq 0$. We define $t_{1}$ as a minimum point of $r_{k}$, that is, $r_{k}\left(t_{1}\right)=$ $\inf _{t \in T} r_{k}(t)$ (we use the fact that $(T, d)$ is compact). Then we define an open subset $A_{1}$ in $T$ by

$$
A_{1}:=\left\{s \in T: 2\left(r_{k}(s)+r_{k}\left(t_{1}\right)\right)>d\left(s, t_{1}\right)\right\} .
$$

Suppose we have constructed points $t_{1}, \ldots, t_{l}$ and open sets $A_{1}, \ldots, A_{l}$. If $T \backslash \bigcup_{j=1}^{l} A_{j}$ is non-empty, then we define $t_{l+1}$ as a minimum point of $r_{k}$ on this set (which is again compact), and set

$$
A_{l+1}:=\left\{s \in T: 2\left(r_{k}(s)+r_{k}\left(t_{l+1}\right)\right)>d\left(s, t_{l+1}\right)\right\} .
$$

Note that by the definition $d\left(t_{j}, t_{l}\right) \geq 2\left(r_{k}\left(t_{j}\right)+r_{k}\left(t_{l}\right)\right)$ if $j \neq l$, and hence $B\left(t_{j}, r_{k}\left(t_{j}\right)\right)$ and $B\left(t_{l}, r_{k}\left(t_{l}\right)\right)$ are disjoint. It follows that

$$
1=m(T) \geq \sum_{j=1}^{\left|T_{k}\right|} m\left(B\left(t_{j}, r_{k}\left(t_{j}\right)\right)\right) \geq \frac{\left|T_{k}\right|}{N_{k}} .
$$

Thus $\left|T_{k}\right| \leq N_{k}$, which implies that our construction stops after a finite number of steps. Clearly $N_{0}=1$ implies that $\left|T_{0}\right|=1$. For each $t \in T$ there exists the smallest $l=l_{0}$ such that $t \in A_{l}$. By the construction we have $2\left(r_{k}(t)+r_{k}\left(t_{l_{0}}\right)\right)>d\left(t, t_{l_{0}}\right)$ and $r_{k}\left(t_{l_{0}}\right) \leq r_{k}(t)$, hence $d\left(t, T_{k}\right)<4 r_{k}(t)$.

To prove the last assertion we consider $x \in B\left(t_{l_{0}}, r_{k}\left(t_{l_{0}}\right)\right)$ with $t_{l_{0}} \in T_{k}$. There exists the smallest $l=l_{1}$ such that $x \in A_{l}$; if $l_{0} \leq l_{1}$ then $r_{k}\left(t_{l_{0}}\right) \leq$ $r_{k}\left(t_{l_{1}}\right) \leq r_{k}(x)$, which ends the proof in this case. If $l_{1}<l_{0}$, then $t_{l_{0}} \notin$ $\bigcup_{j=1}^{l_{1}} A_{j}$ and so $d\left(t_{l_{0}}, t_{l_{1}}\right) \geq 2\left(r_{k}\left(t_{l_{0}}\right)+r_{k}\left(t_{l_{1}}\right)\right)$. Consequently, by the triangle inequality,

$$
2\left(r_{k}\left(t_{l_{0}}\right)+r_{k}\left(t_{l_{1}}\right)\right) \leq d\left(t_{l_{0}}, t_{l_{1}}\right) \leq d\left(x, t_{l_{1}}\right)+d\left(x, t_{l_{0}}\right) \leq d\left(x, t_{l_{1}}\right)+r_{k}\left(t_{l_{0}}\right),
$$

where the last inequality follows because $x \in B\left(t_{l_{0}}, r_{k}\left(t_{l_{0}}\right)\right)$. On the other hand, $x \in A_{l_{1}}$, so

$$
d\left(x, t_{l_{1}}\right)<2\left(r_{k}(x)+r_{k}\left(t_{l_{1}}\right)\right) .
$$

It follows that 


$$
2\left(r_{k}\left(t_{l_{0}}\right)+r_{k}\left(t_{l_{1}}\right)\right) \leq d\left(x, t_{l_{1}}\right)+r_{k}\left(t_{l_{0}}\right) \leq 2\left(r_{k}(x)+r_{k}\left(t_{l_{1}}\right)\right)+r_{k}\left(t_{l_{0}}\right),
$$

and hence $r_{k}\left(t_{l_{0}}\right) \leq 2 r_{k}(x)$ as desired.

Proof of Theorem 3. By Theorem 4 there exists an admissible net $\mathcal{T}=$ $\left(T_{k}\right)_{k>0}$ such that $d\left(t, T_{k}\right) \leq 4 r_{k}(t)$. Consequently, Lemma 3 shows that for each $t \in T$ we have

$$
\begin{aligned}
\sum_{k=0}^{\infty} d\left(t, T_{k}\right) \varphi^{-1}\left(N_{k}\right) & \leq 4 \sum_{k=0}^{\infty} r_{k}(t) \varphi^{-1}\left(N_{k}\right) \\
& \leq \frac{4 c}{c-1} \int_{0}^{D(t, T)} \varphi^{-1}\left(\frac{1}{m(B(t, \varepsilon))}\right) d \varepsilon,
\end{aligned}
$$

which implies that

$$
\sup _{t \in T} \sum_{k=0}^{\infty} d\left(t, T_{k}\right) \varphi^{-1}\left(N_{k}\right) \leq \frac{4 c}{c-1} \mathcal{M}(m, \varphi) .
$$

To show the second claim we first check that since $1 / N_{k+1} \leq m\left(B\left(t, r_{k+1}(t)\right)\right)$ and $d\left(t, T_{k}\right) \leq 4 r_{k}(t)$ one can see that

$$
\sum_{t \in T_{k+1}} \frac{d\left(t, T_{k}\right) \varphi^{-1}\left(N_{k}\right)}{N_{k+1}} \leq 4 \sum_{t \in T_{k}} \int_{B\left(t, r_{k+1}(t)\right)} r_{k}(t) \varphi^{-1}\left(N_{k}\right) m(d x) .
$$

By the Lipschitz property of $r_{k}$ (Lemma 2) we derive that $r_{k}(t) \leq r_{k}(x)+$ $r_{k+1}(t)$ for $x \in B\left(t, r_{k+1}(t)\right)$. Therefore

$$
\int_{B\left(t, r_{k+1}(t)\right)} r_{k}(t) \varphi^{-1}\left(N_{k}\right) m(d x) \leq \int_{B\left(t, r_{k+1}(t)\right)}\left(r_{k}(x)+r_{k+1}(t)\right) \varphi^{-1}\left(N_{k}\right) m(d x) .
$$

The last assertion in Theorem 4 implies that $r_{k+1}(t) \leq 2 r_{k+1}(x)$ for any $t \in T_{k+1}$ and $x \in B\left(t, r_{k+1}(t)\right)$. Hence

$$
\int_{B\left(t, r_{k+1}(t)\right)} r_{k}(t) \varphi^{-1}\left(N_{k}\right) m(d x) \leq \int_{B\left(t, r_{k+1}(t)\right)}\left(r_{k}(x)+2 r_{k+1}(x)\right) \varphi^{-1}\left(N_{k}\right) m(d x) .
$$

Since $B\left(t, r_{k+1}(t)\right)$ are disjoint for $t \in T_{k}$ (the second claim in Theorem 4), we derive

$$
\sum_{t \in T_{k}} \int_{B\left(t, r_{k+1}(t)\right)} r_{k}(t) \varphi^{-1}\left(N_{k}\right) m(d x) \leq 2 \int_{T}\left(r_{k}(x)+2 r_{k+1}(x)\right) \varphi^{-1}\left(N_{k}\right) m(d x) .
$$

Combining the above inequality with (12) and (3) (with $c>2$ ) we deduce that

$$
\sum_{k=0}^{\infty} \frac{d\left(t, T_{k}\right) \varphi^{-1}\left(N_{k}\right)}{N_{k+1}} \leq 4 \int \sum_{T} \sum_{k=0}^{\infty} r_{k}(x) \varphi^{-1}\left(N_{k}\right) m(d x) .
$$


It remains to use Lemma 3, which yields

$$
\sum_{k=0}^{\infty} \frac{d\left(t, T_{k}\right) \varphi^{-1}\left(N_{k}\right)}{N_{k+1}} \leq \frac{4 c}{c-1} \overline{\mathcal{M}}(m, \varphi) .
$$

\section{References}

[1] W. Bednorz, A theorem on majorizing measures, Ann. Probab. 34 (2006), 1771-1781.

[2] X. Fernique, Régularité des trajectoires des fonctions aléatoires gaussiennes, in: École d'Été de Probabilités de Saint-Flour, IV-1974, Lecture Notes in Math. 480, Springer, Berlin, 1975, 1-96.

[3] M. Talagrand, Sample boundedness of stochastic processes under increment conditions, Ann. Probab. 18 (1990), 1-49.

[4] -, Majorizing measures without measures, ibid. 29 (2001), 411-417.

[5] -, The Generic Chaining. Upper and Lower Bounds of Stochastic Processes, Springer, Berlin, 2005.

Witold Bednorz

Department of Mathematics

University of Warsaw

Banacha 2

02-097 Warszawa, Poland

E-mail: wbednorz@mimuw.edu.pl

Received October 3, 2007;

received in final form May 16, 2008 